\title{
Thermal modeling environment for TMT
}

\author{
Konstantinos Vogiatzis \\ TMT Observatory Corporation, Pasadena, CA 91125, USA
}

\begin{abstract}
In a previous study we had presented a summary of the TMT Aero-Thermal modeling effort to support thermal seeing and dynamic loading estimates. In this paper a summary of the current status of Computational Fluid Dynamics (CFD) simulations for TMT is presented, with the focus shifted in particular towards the synergy between CFD and the TMT Finite Element Analysis (FEA) structural and optical models, so that the thermal and consequent optical deformations of the telescope can be calculated.

To minimize thermal deformations and mirror seeing the TMT enclosure will be air conditioned during day-time to the expected night-time ambient temperature. Transient simulations with closed shutter were performed to investigate the optimum cooling configuration and power requirements for the standard telescope parking position.

A complete model of the observatory on Mauna Kea was used to calculate night-time air temperature inside the enclosure (along with velocity and pressure) for a matrix of given telescope orientations and enclosure configurations. Generated records of temperature variations inside the air volume of the optical paths are also fed into the TMT thermal seeing model.

The temperature and heat transfer coefficient outputs from both models are used as input surface boundary conditions in the telescope structure and optics FEA models. The results are parameterized so that sequential records several days long can be generated and used by the FEA model to estimate the observing spatial and temporal temperature range of the structure and optics.
\end{abstract}

Keywords: Extremely Large Telescopes, Thermal Modeling, Computational Fluid Dynamics

\section{INTRODUCTION}

Thermal Management is a key aspect of Wave-front Control Architecture. Approximately $30 \%$ of the total seeing limited error budget allocation has aero-thermal causes, with $80 \%$ of that attributed to thermal seeing and deformations. As tools to minimize thermal effects TMT uses air-conditioning during day-time, passive ventilation during night-time and heat release control in various forms (sub-system cooling, proper location of heat sources, coatings of desired emissivity). The optimization of the configuration and operation of these tools is done by modeling and is beyond the scope of this paper. However, once these tools have been incorporated in the design, they create a more stable thermal environment for the telescope structure and the optics and different active optics correction scenarios can be modeled.

Figure 1 depicts the current TMT aero-thermal modeling synergy. The gray box called "Stochastic Framework" represents the TMT Monte Carlo simulation test-bed [1] and fuses together performance estimates for optical surface shapes, wind disturbances and thermal seeing. It utilizes the normalized Point Source Sensitivity (PSSn) performance metric [2]. A 3-year environmental record [3] measured on 13N, Mauna Kea, the TMT site, provides ambient condition variability and the corresponding telescope pointing record from Gemini North provides orientation statistics. The CFD models provide heat transfer coefficients to the Segment Support Assembly (SSA), M2/M3 and telescope structure FE solid thermal models $[4,5]$, which in turn provide temperature and heat fluxes to the CFD models as boundary conditions for the telescope and optical surfaces. The surface thermal model provides heat fluxes to the CFD models for the enclosure surfaces. It can also provide the FEA models with correct radiation properties. The convective heat transfer coefficients needed by the surface model are provided by isothermal CFD simulations. Finally the air-conditioning model provides fast qualitative information about optimum day-time thermal control strategies to minimize the computationally intensive day-time CFD simulations. The thermal CFD simulations yield velocity and temperature fields used by the wind [6] and thermal seeing [7] models respectively. The thermal FEA simulations yield displacements used by the optical aberrations model [8]. The TMT Merit Function Routine [9] can also act as an estimator of optical aberrations caused by structural deformations, including gravity induced ones.

In the following sections we will present a summary of the CFD day-time and night-time simulations performed to date. Then the philosophy behind generating a realistic input environment for the FEA simulations will be explained. 


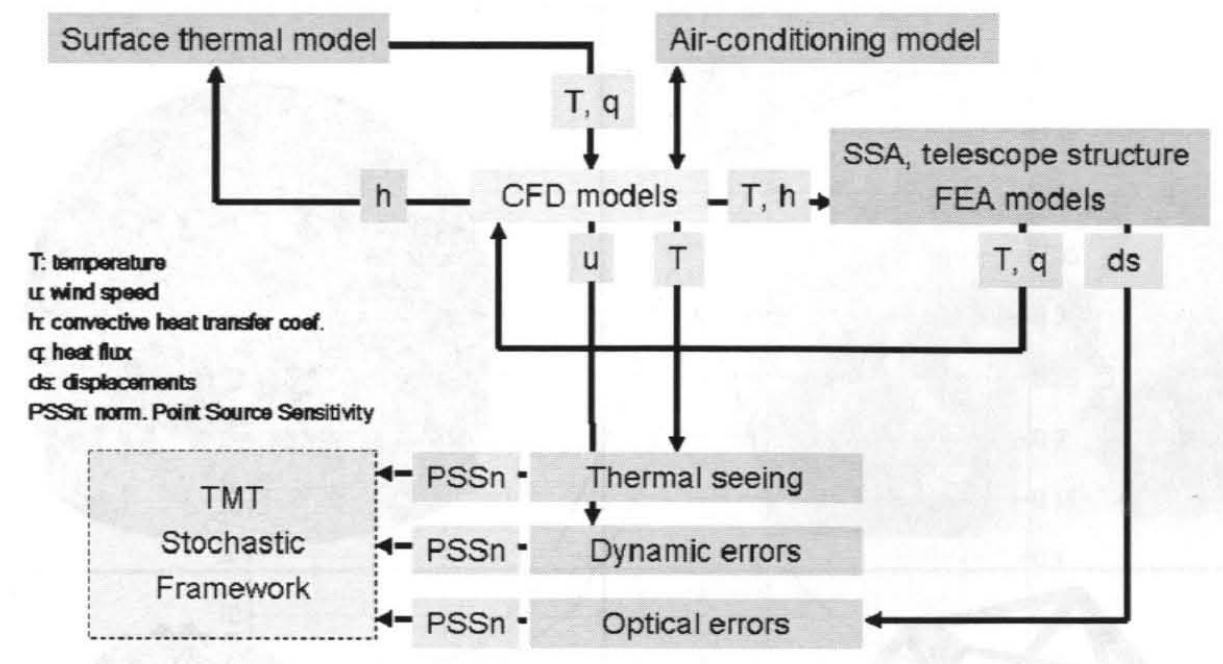

Figure 1 TMT aero-thermal modeling information flow-chart

\section{NIGHT-TIME SIMULATIONS}

The general set-up for CFD simulations has been extensively reported in earlier work $[10,11]$. We will focus here on the enhancements-updates. Figure 2 shows the latest TMT enclosure and telescope design resolved by CFD. The Calotte enclosure is placed on the $13 \mathrm{~N}$ site on Mauna Kea and the facility building has been incorporated. The topographic DEM has a $10 \mathrm{~m}$ resolution while the enclosure and telescope grids have a horizontal surface resolution of $0.5 \mathrm{~m}-1 \mathrm{~m}$ and a surface thickness resolution of $0.01 \mathrm{~m}-0.05 \mathrm{~m}$. Features that do not affect the flow aerodynamically or thermally and are less than $0.1 \mathrm{~m}$ in diameter have been omitted. The computational domain is $3400 \mathrm{~m} \mathrm{E}-\mathrm{W}, 2500 \mathrm{~m} \mathrm{~N}-\mathrm{S}$ and $\sim 700 \mathrm{~m}$ in height. The enclosure interstitial space is now resolved. The secondary support structure and tripod were also added. The M1 is segmented without gaps, with 48 equilateral cells per segment ( 24 on either side). 492 "SSA" prisms have been added with 20 cells each and 84 node boxes with 12 cells each.

Three different orientations have been investigated so far, for a zenith angle of $32.5^{\circ}$, relative to wind azimuth angles of $0^{\circ}, 90^{\circ}, 180^{\circ}$, and vents being fully open, half open and fully closed. The relative to wind azimuth angle is defined by the wind direction and the telescope plane normal to the elevation axis. Air properties have been taken at $\sim 4000 \mathrm{~m}$. The ground temperature is set to $0.5 \mathrm{~K}$ below ambient reference temperature. The latter is set at the median night-time level of $276 \mathrm{~K}$. For the closed vents cases the reference wind speed at $20 \mathrm{~m}$ is $\sim 10 \mathrm{~m} / \mathrm{s}$ and for the open vent cases is $\sim 2.5 \mathrm{~m} / \mathrm{s}$. For the half open vent cases it is $\sim 6.5 \mathrm{~m} / \mathrm{s}$. In all cases the average resulting wind speed above the primary mirror is $\sim 0.5$ $1.5 \mathrm{~m} / \mathrm{s}$. The current TMT M1 target velocity is approximately $1 \mathrm{~m} / \mathrm{s}$.

The CFD simulations performed can be grouped into two categories, steady state and transient (or unsteady).

Steady state simulations are used to provide the wind reduction factors for telescope top end and segment wind buffeting in the form of mean and rms velocities, normalized with the reference wind speed. They also provide information on venting efficiency for mirror flushing and enclosure volume exchange. Convective heat transfer coefficient maps on the telescope and on the enclosure surfaces are also provided for use in the FEA and thermal models respectively. Finally the simulations are used to provide feedback to summit development design.

Unsteady simulations are useful for high order wind and thermal behavior. Temperature records inside the optical path are provided to the thermal seeing models. Velocity and pressure records and spectra on the telescope or the enclosure exterior surfaces can also be used in the wind loading models. New thermal seeing models have been developed that can in fact use steady state results, in the form of temperature, turbulent energy dissipation rate and turbulent viscosity fields. The transient simulations are still, however, used for bench-marking the wind loading and thermal seeing models. 


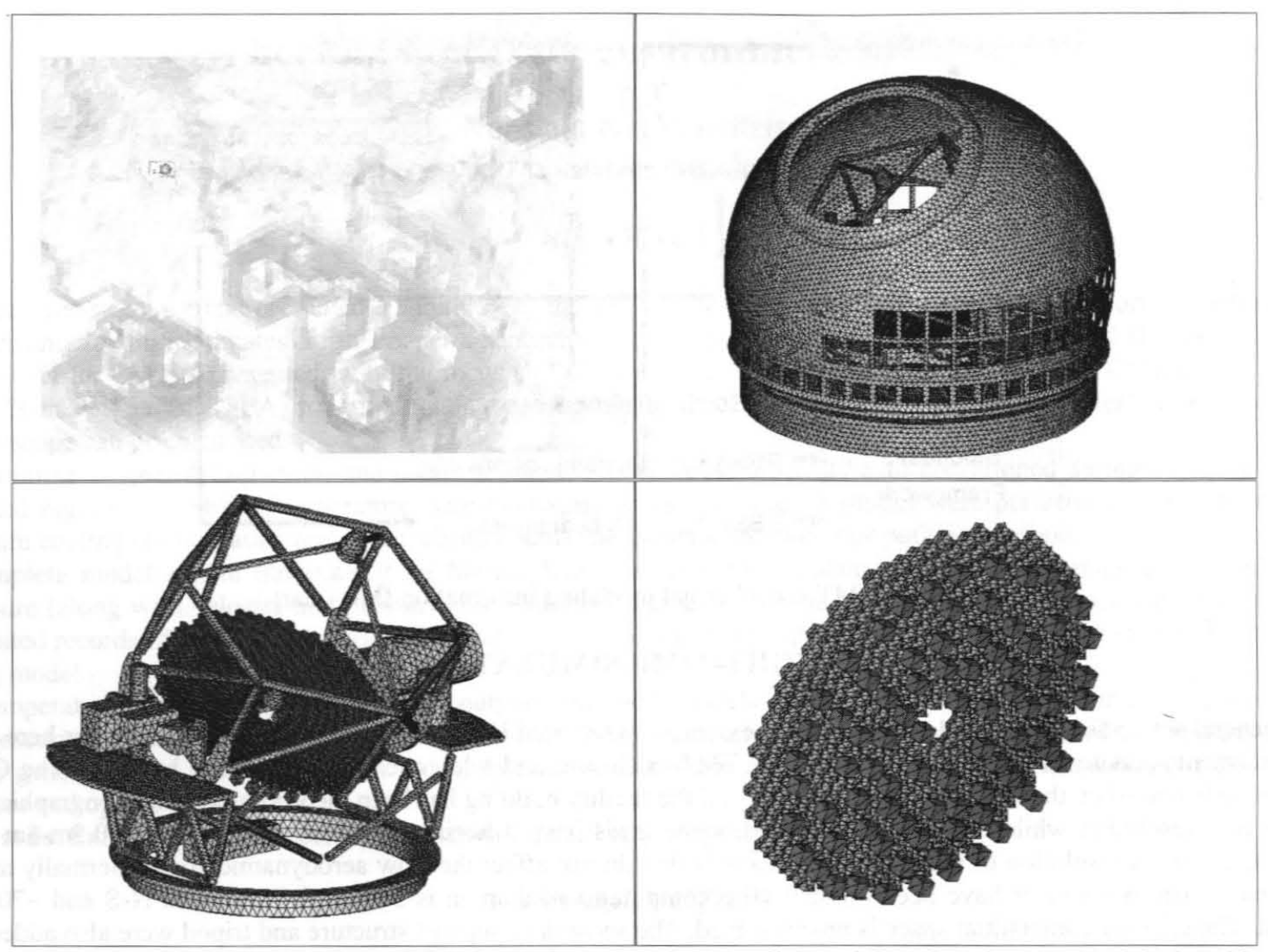

Figure 2 Mauna Kea CFD model (top left), the enclosure surface detail (top right), the telescope model placed inside (bottom left) and grid detail behind M1 (bottom right)

\section{DAY-TIME SIMULATIONS}

It is prudent to minimize the difference between the temperature of the telescope (structure and optics) and the average expected temperature during the night. Especially for the optics, given their larger time constant, this is important both for mitigating thermal deformation and mirror seeing. In addition, spatial temperature gradients should be minimized since they also are responsible for telescope misalignment.

Figure 3 shows the distribution of the expected difference between the temperature at sunrise and the average temperature of the next night, based on the 3-year period of TMT site testing on Mauna Kea. It is assumed that at sunrise the enclosure will close and the air trapped inside will start warming up due to day-time heat release and solar radiation. Ideally we would like to be able to rapidly bring the bulk air temperature close to the night-time average $\left(\mathrm{T}_{\text {target }}\right)$ while minimizing any vertical gradients. This will help the telescope structure and optics oscillate within a small temperature range and drift away from the conditions of the last APS calibration at a slow rate. From the figure, we can see that $70 \%$ of the time we should expect to change the bulk temperature of the enclosure by $\pm 1.5 \mathrm{~K}$. The median temperature difference is $+0.5 \mathrm{~K}$ but we will investigate both cases, where the target temperature is $1.5 \mathrm{~K}$ higher and $1.5 \mathrm{~K}$ lower than the sunrise temperature. A third case, where the target temperature is $1.5 \mathrm{~K}$ higher than the sunrise but there is no cooled air supplied to the enclosure will also be presented. 


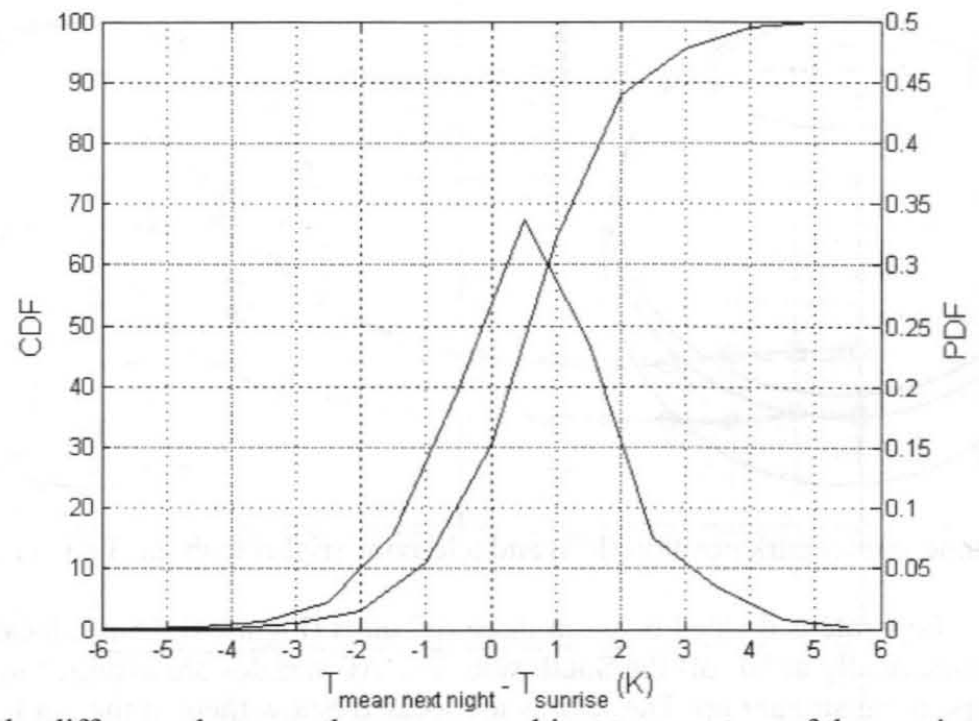

Figure 3 Distribution of the difference between the average ambient temperature of the coming night and the ambient temperature at sunrise

CFD simulations were performed for these cases for a nominal day of $12 \mathrm{~h}$. Table 1 summarizes the case configurations, while more details are presented in the Appendix. The solar flux has a sinusoidal form and corresponds to median wind and ambient temperature conditions and a $5 \mathrm{~K}$ diurnal variation.

Table 1 Air conditioning cases configuration

\begin{tabular}{|c|c|c|c|}
\hline Case & $\# \mathbf{1}$ & $\mathbf{\# 2}$ & $\# \mathbf{3}$ \\
\hline $\mathrm{T}_{\text {target }}(\mathrm{K})$ & 275.5 & 275.5 & 275.5 \\
\hline $\mathrm{T}_{\text {sunrise }}(\mathrm{K})$ & 274 & 277 & 274 \\
\hline $\mathrm{T}_{\text {nozzle }}(\mathrm{K})$ & 273 & 273 & $\mathrm{~T}_{\text {sunrise }}+5 \sin (\boldsymbol{\pi} \mathrm{t} /$ day $)$ \\
\hline AC air flow rate $(\mathrm{cfm})$ & 51600 & 51600 & 51600 \\
\hline Top venting $(\mathrm{cfm})$ & 10300 & 10300 & 10300 \\
\hline Internal heat load $(\mathrm{W})$ & 40000 & 40000 & 40000 \\
Zenith solar flux $\left(\mathrm{W} / \mathrm{m}^{2}\right)$ & 2.3 & 2.3 & 2.3 \\
\hline
\end{tabular}

For case 3 it is assumed that ambient air replaces the cooled air the AC system would provide. It is also assumed that there will be a relief vent at the top of the dome. The vent is assumed rectangular with an approximate area of $1 \mathrm{~m}^{2}$. Due to maintenance considerations the vent is assumed to be located near the cap-base interface. Ideally it would be extracting air for the highest point of the dome. This requires additional ductwork which is not part of the current design but can be easily implemented. However, the simulation assumed that that would be the case. The air temperature above the vent level if it is not located at the maximum height is expected to be $10 \mathrm{~K}-15 \mathrm{~K}$ higher. The shutter is facing North at the $65^{\circ}$ zenith position (Figure 4). The telescope is parked at $90^{\circ}$ zenith, pointing to the South. 


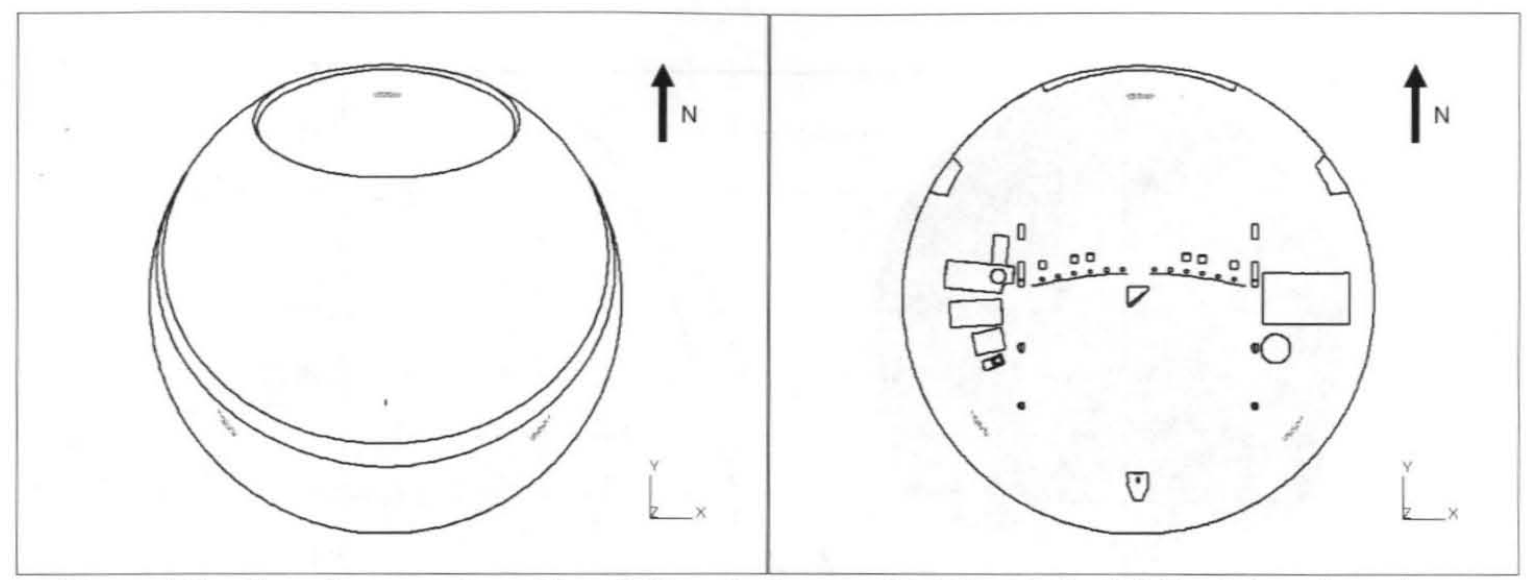

Figure 4 Daytime shutter park position (left) and telescope (right) with the $3 \mathrm{AC}$ unit locations shown

For the AC cases, the air flow rate is divided between three AC units (Figure 4). One is located on the North side of the fixed base, and two symmetrically at $50^{\circ}$ off the South side. The AC nozzles are assumed to supply air to the enclosure interior at the $7 \mathrm{~m}$ level pointing straight up. The returns are located below them at the $3 \mathrm{~m}$ level. Note that during initial simulations the nozzles were pointing at an angle with respect to vertical and with respect to the center of the dome creating a swirling motion to enhance mixing. Mixing occurs naturally aided by the curvature of the dome after $\sim 30 \mathrm{~m}$ of height and the fact that eventually the flow has to come down to the returns. Swirling can also occur if the returns are not positioned directly under the nozzles, but this is harder to implement.

Figures 5, 6 and 7 show the resulting temporal behavior of the air temperature inside the dome at two different levels, $20 \mathrm{~m}$ from the floor and $38 \mathrm{~m}$, top of the primary mirror. The target and provided air temperatures are also included. Figure 8 summarizes the sunset air temperature profiles inside the enclosure for all three cases.

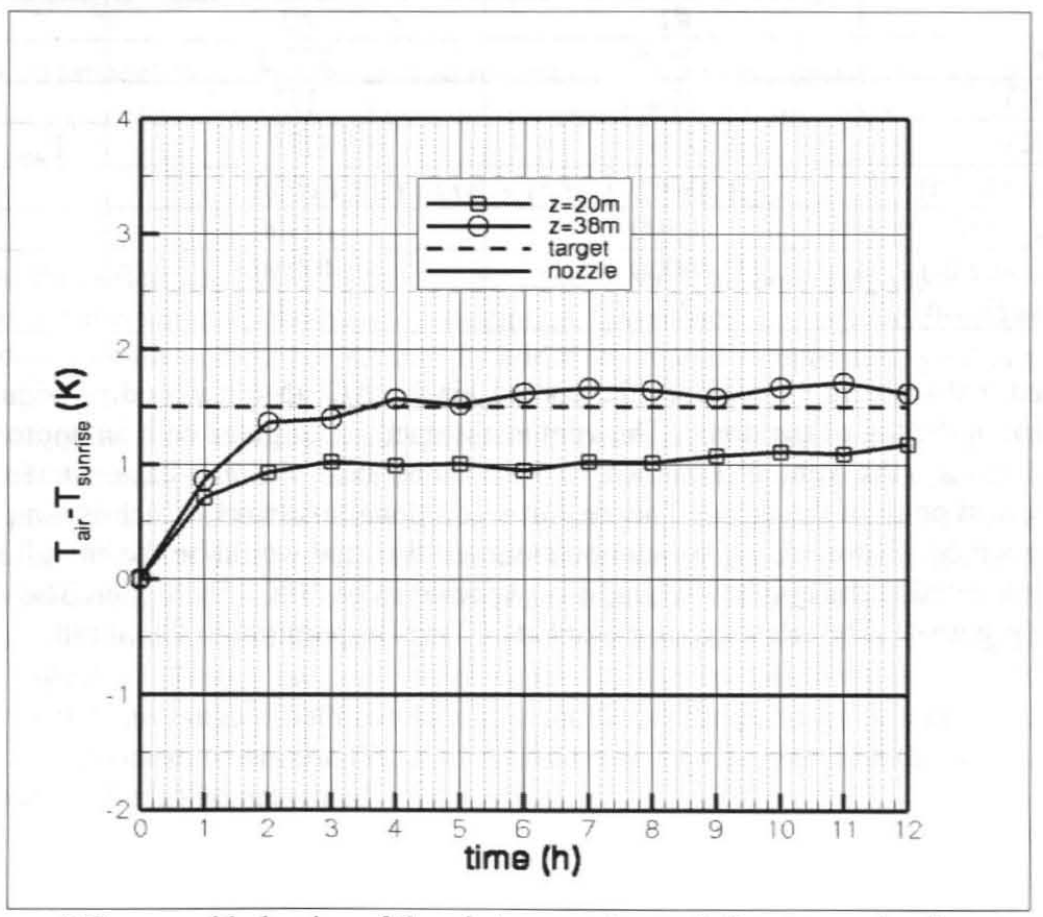

Figure 5 Temporal behavior of the air temperature relative to sunrise for case \#1 


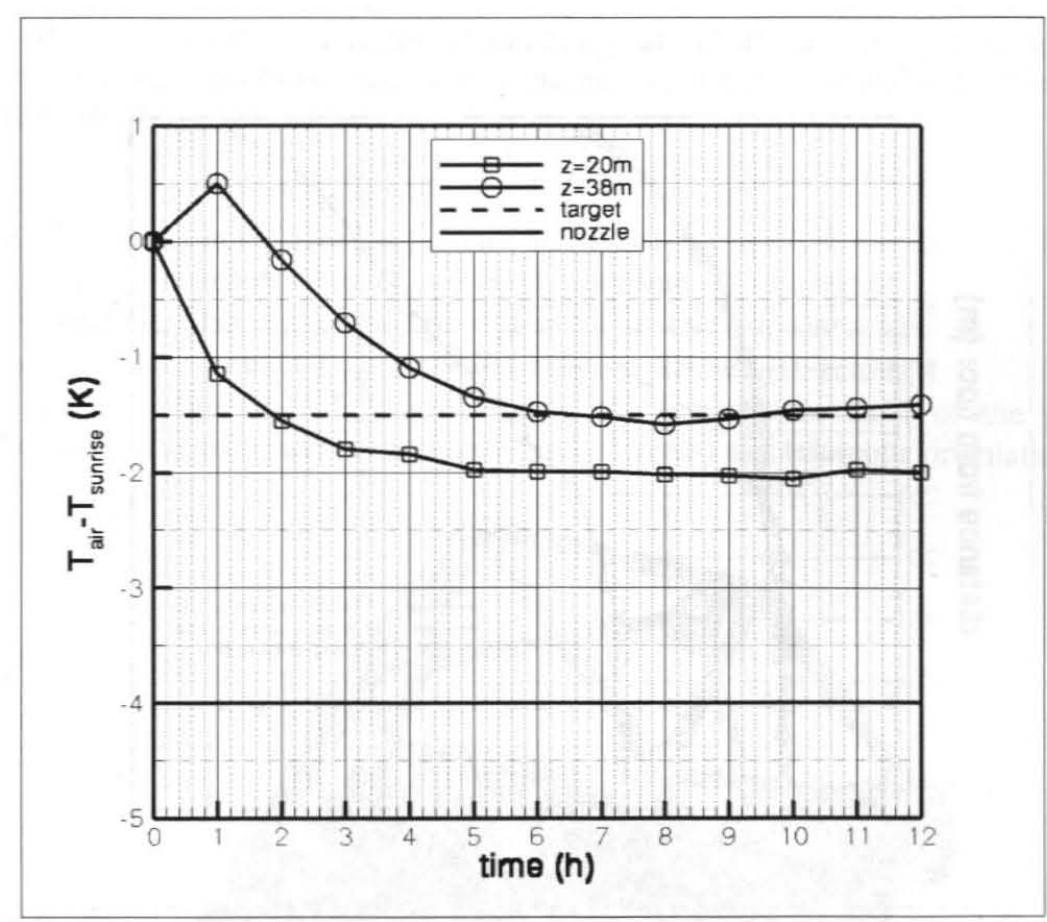

Figure 6 Temporal behavior of the air temperature relative to sunrise for case $\# 2$

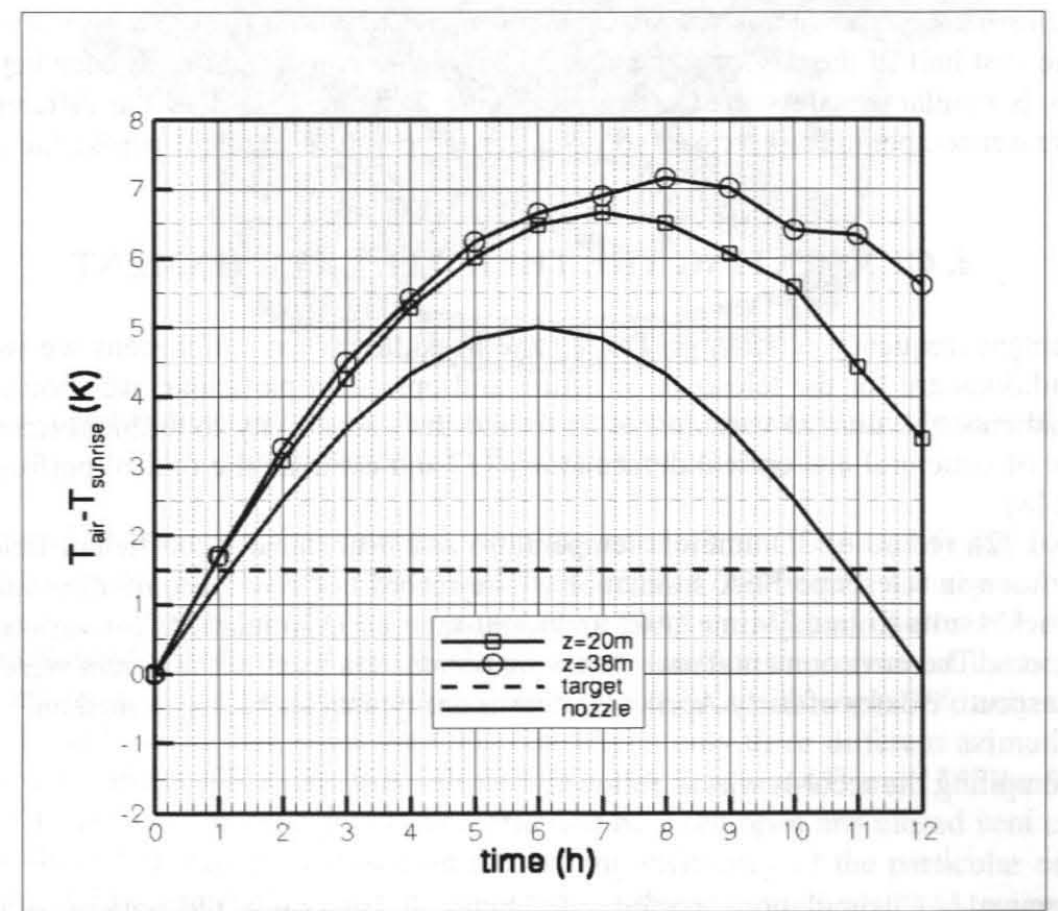

Figure 7 Temporal behavior of the air temperature relative to sunrise for case \#3 


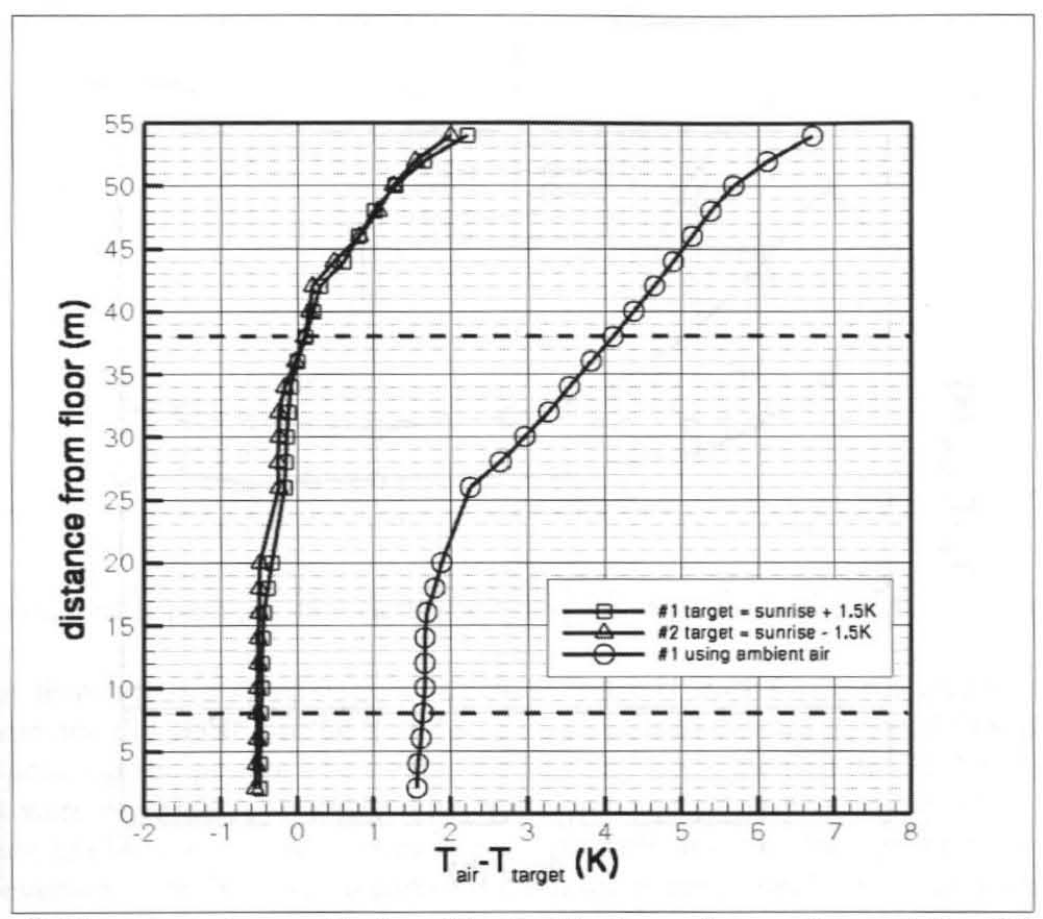

Figure 8 Air temperature vertical profiles inside the enclosure at sunset for all 3 cases

Since naturally the enclosure air will become warmer, it takes longer to cool it than to let it warm up "gracefully", but it still happens within the first half of the day. At the end of the day the temperature difference between the two locations for the first two cases is similar and less than a degree. Figure 8 suggests that as the difference between the target temperature and the sunset temperature increases so does the temperature gradient across the mirror, so the negative impact is two-fold.

\section{GENERATING THE THERMAL ENVIRONMENT}

APS nominal measurement frequency is 15 days. During the period between calibrations we would like to know how stable the thermal conditions are for the telescope structure and optics. In particular, we should estimate the expected spatial temperature gradients, the diurnal variation of them and their sensitivity to ambient temperature changes. Then we can obtain a record of structural and optical deformations [5] and estimate the optical performance consequences of telescope thermal drift [8].

To that effect, an initial $72 \mathrm{~h}$ record of $3 \mathrm{D}$ ambient temperature and heat transfer coefficient fields was generated to be used as boundary condition in telescope FEA simulations. The record has now become the standard telescope thermal environment. It combines results from day-time (AC) and night-time CFD simulations for various telescope orientations and venting configurations. The environmental and observing parameters used in the model were extracted from [3] and correspond to three consecutive dates of early April. Conditions are typical and close to median.

The methodology of compiling the records was as follows:

\section{Day-time}

- From the two nominal AC simulations performed, obtain $\mathrm{T}_{\text {air }}(\mathrm{z})$ (since the pattern is stratified, every $5 \mathrm{~m}$ is sufficient) and $\mathrm{T}_{\text {target }}-\mathrm{T}_{\text {sunrise }}$ as well as $\mathrm{T}_{\text {target }}-\mathrm{T}_{\mathrm{AC}}$

- Obtain heat transfer coefficient $\mathrm{h}$ on several regions of the telescope structure, nominally every $5 \mathrm{~m}$

- Parameterize $T_{\text {air }}(\mathrm{z}, \mathrm{t})$ to account for different $\mathrm{T}_{\text {sunrise }}, \mathrm{T}_{\text {target }}$ and $\mathrm{T}_{\text {nozzle }}$ 
- The day-time record is $12.5 \mathrm{~h}$ long and temporal sampling rate of $1 \mathrm{~h}$ is sufficient. In general $\mathrm{T}_{\text {target }}$ can be the expected mean of the coming night (since relatively accurate forecasting is available for Mauna Kea) and $\mathrm{T}_{\text {nоzzle }}$ will be typically $2 \mathrm{~K}-3 \mathrm{~K}$ below $\mathrm{T}_{\text {target }}$.

\section{Night-time}

- From the various CFD simulations performed, obtain $\mathrm{T}_{\text {air }}(\mathrm{x}, \mathrm{y}, \mathrm{z})-\mathrm{T}_{\text {ref }}$ (upwind) for each configuration; $\mathrm{T}_{\text {ref }}$ should be offset if necessary to make it compatible to $\mathrm{T}_{\text {target }}$ from the daytime $\mathrm{AC}$ simulation

- Obtain heat transfer coefficient $\mathrm{h}(\mathrm{x}, \mathrm{y}, \mathrm{z})$ on several regions of the telescope structure

- From the environmental record, extract $T_{\text {ambient }}(t), \mathrm{U}(\mathrm{t})$, relative_azimuth $(\mathrm{t})$, zenith $(\mathrm{t})$

- Set $\mathrm{T}_{\text {ref }}$ equal to $\mathrm{T}_{\text {ambient }}(\mathrm{t})$, generate $\mathrm{T}_{\text {air }}(\mathrm{x}, \mathrm{y}, \mathrm{z}, \mathrm{t})$ using the temperature field of the particular configuration compatible with the instantaneous wind speed (open or closed vents) and telescope orientation

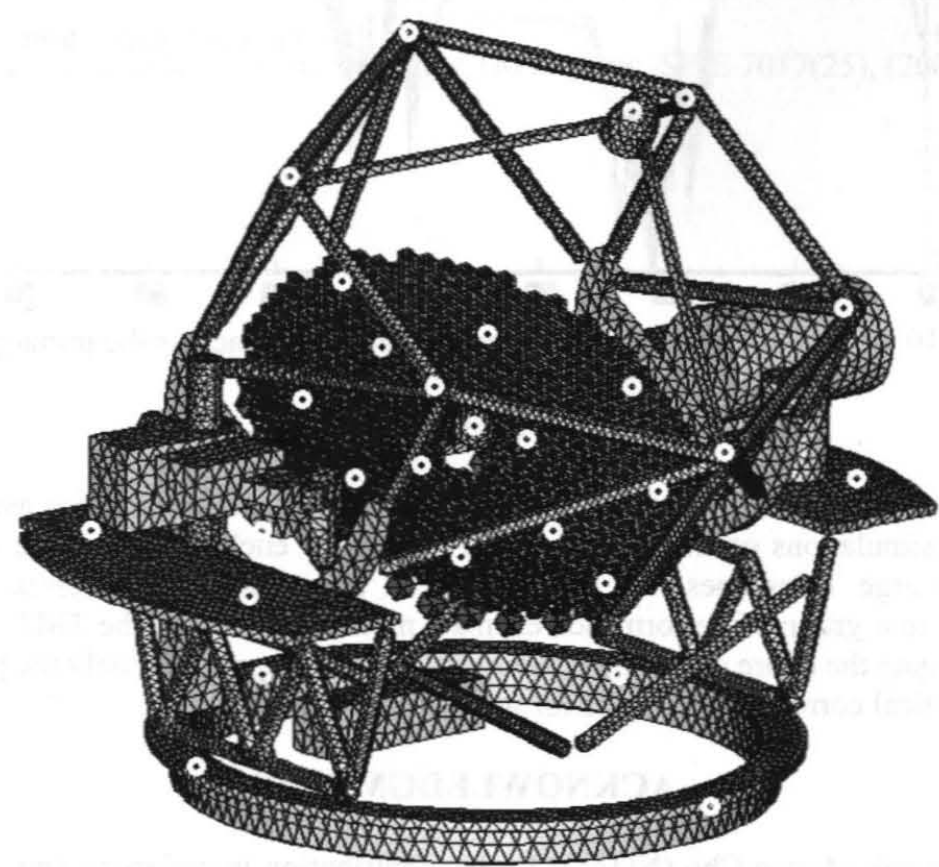

Figure 9 Telescope locations (white circles) where ambient temperature and heat transfer coefficient are reported for FEA boundary conditions

The night-time record is $11.5 \mathrm{~h}$ long and the temporal sampling rate is $2 \mathrm{~min}$. To simplify the FEA inputs, 30 locations were identified on the telescope, where temperatures and heat transfer coefficients are reported. Figure 9 shows their distribution. They consist of 12 points on M1, one representative point on M2 and one on M3, one for each of the two elevation rings, 4 on the Nasmyth platforms, 4 on the telescope pier and 6 on the corners of the hex ring.

Since the FEA night-time model is so far available only for a telescope zenith angle $30^{\circ}$, only the corresponding CFD cases were considered. In addition to that, since the CFD considered only three different azimuth angles, if the relative azimuth angle was below $45^{\circ}$ the $0^{\circ} \mathrm{CFD}$ case was selected, if it was between $45^{\circ}$ and $135^{\circ}$ the $90^{\circ} \mathrm{CFD}$ case was used and above $135^{\circ}$ the $180^{\circ} \mathrm{CFD}$ case was considered. As a criterion between open and closed vent cases the resulting wind speed cut-off of $\sim 1 \mathrm{~m} / \mathrm{s}$ above M1 was used, based on the venting efficiency of the particular orientation. It should be noted that the night-time CFD flow fields used in this study still correspond to the Armazones site in Chile. The environmental record will be updated in the near future.

The resulting ambient air temperature records for the entire $72 \mathrm{~h}$ period is depicted in Figure 10 for 5 levels along the $\mathrm{Y}$ TMT local telescope axis, with X being the Elevation Axis. The levels are $5 \mathrm{~m}$ apart and cover the $30 \mathrm{~m}$ of the primary mirror cell for both day- and night-time. In this study day-time $\mathrm{AC} \mathrm{T}_{\text {target }}$ was set to be the mean of the previous night, which required the environmental record of the night preceding the $72 \mathrm{~h}$ environmental record. This also has the potential of missing the target for the last night as can be seen in Figure 10. Both options can be studied with the FEA modeling. 


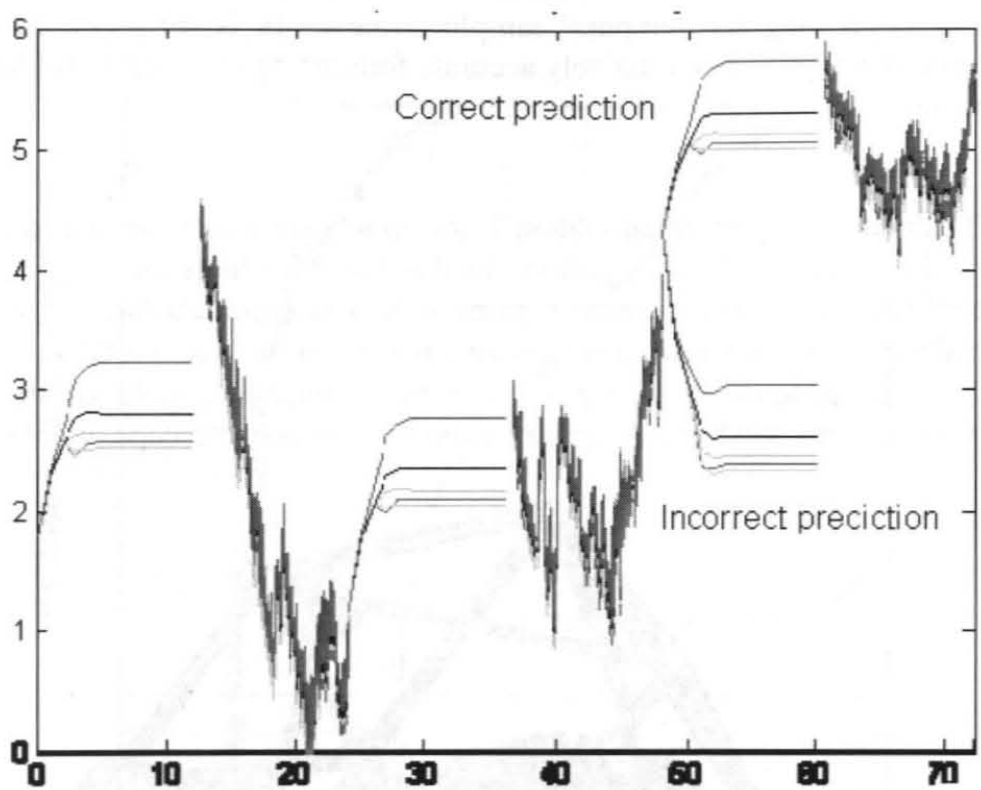

Figure 10 Record of ambient air temperatures at 5 levels across the primary mirror

\section{CONCLUSIONS}

The brief overview of results presented here provides only a sample of the capabilities available through the current thermal models and CFD simulations of wind flow around and inside enclosure-telescope configurations for the next generation of Extremely Large Telescopes. We have enhanced the existing aero-thermal modeling framework that provides a range of inputs to a group of performance estimate models utilized by the TMT Systems Engineering team. We are now able to investigate the entire operating envelope in order to support not only the phase of detailed design but also the development of optical correction strategies for wave-front control.

\section{ACKNOWLEDGMENTS}

The author would like to thank Myung Cho (NOAO) for his contribution in understanding the thermal environment of the TMT primary mirror.

The author gratefully acknowledges the support of the TMT partner institutions. They are the Association of Canadian Universities for Research in Astronomy (ACURA), the California Institute of Technology and the University of California. This work was supported as well by the Gordon and Betty Moore Foundation, the Canada Foundation for Innovation, the Ontario Ministry of Research and Innovation, the National Research Council of Canada, the Natural Sciences and Engineering Research Council of Canada, the British Columbia Knowledge Development Fund, the Association of Universities for Research in Astronomy (AURA) and the U.S. National Science Foundation.

\section{REFERENCES}

[1] Angeli, G. Z., Roberts, S. and Vogiatzis, K., "Systems Engineering for the Thirty Meter Telescope", SPIE 7738(45), (2010).

[2] Seo, B.-J., Nissly, C. R., Angeli, G. Z., Ellerbroek, B., Nelson, J., Sigrist, N. and Troy, M., "Analysis of normalized point source sensitivity as a performance metric for large telescopes", Applied Optics, vol. 48, no 31, pp. 5997-6007, (2009).

[3] Schöck, M. et al, "Status of the Thirty Meter Telescope site selection program”, Proc. SPIE 7012(68), (2008).

[4] Cho, M., Corredor, A., Pootrakul, S. and Vogiatzis, K., "Thermal performance prediction of the TMT optics", Proc. SPIE 7017(43), (2008). 
[5] Cho, M., Corredor, A.,Vogiatzis, K. and Angeli, G. Z., "Thermal analysis of the TMT telescope structure", SPIE 7738(12), (2010).

[6] MacMynowski, D. G., Colavita, M. M., Skidmore, W. A. and Vogiatzis, K., "Primary mirror dynamic disturbance models for TMT: vibration and wind", SPIE 7738(14), (2010).

[7] Pazder, J., Vogiatzis, K., "Dome and mirror seeing estimates for the Thirty Meter Telescope", Proc. SPIE 7017(26), (2008).

[8] Nissly, C. R., Seo, B.-J., Troy, M., Angeli, G. Z., Cho, M., Roberts, Jr., L. C., Shelton, J. C., Sigrist, N., Sirota, M. J. and Stepp, L. M., "Investigation of Thirty Meter Telescope wavefront maintenance using low-order Shack-Hartmann wavefront sensors to correct for thermally induced misalignment", SPIE 7738(17), (2010).

[9] Roberts, S., "Systems Engineering of the Thirty Meter Telescope through integrated opto-mechanical analysis", SPIE 7738(46), (2010).

[10] Vogiatzis, K. and Angeli, G. Z., "Strategies for Estimating Mirror and Dome Seeing for TMT", Proc. SPIE 6271(27), (2006).

[11] Vogiatzis, K., “Advances in aero-thermal modeling for TMT”, Proc. SPIE 7017(25), (2008). 